\title{
RISKS AND OPPORTUNITIES OF USING FIBRE OPTIC SENSORS FOR LONG TERM INFRASTRUCTURE HEALTH MONITORING SYSTEMS IN AN 18 YEAR OLD INSTALLATION
}

\author{
I. Robles Urquijo ${ }^{1}$, A. Quintela Incera ${ }^{2}$, Steven Van Vaerenbergh ${ }^{2}$, D. Inaud ${ }^{3}$ and J.M. Lopez Higuera ${ }^{2}$
}

\author{
${ }^{1}$ WSP (Louis Berger), Santander, Spain \\ ${ }^{2}$ Universidad de Cantabria, Santander, Spain \\ ${ }^{3}$ Smartec, Manno, Switzerland \\ *Corresponding author
}

\begin{abstract}
Uncertainty about the survivability and technological evolution presents its risks when designing the sensors to use on infrastructure health monitoring systems. Fibre optic methods have been available for some time, and are reaching the technological maturity required to provide reliable long-term structural health monitoring solutions. By embedding fibre optic sensors in the structures at construction time, the engineers are able to detect deviations from the structure's original design and expected dynamics. However, due to the long-term infrastructure's life span and the relatively new fibre optic techniques, there are very few historical examples to analyse and use as proof for the adequacy of such techniques to the infrastructure's life-cycle.
\end{abstract}

This study explores the risks and opportunities associated with these technologies through a real case of one of the first Fibre Bragg Grating (FBG) sensorized highway bridge installed in Spain in the year 2000, the «Las Navas» bridge at the A-8 «Autovía del Cantábrico» highway in the north of Spain. The survivability of the fibre optic sensors, after eighteen years of embedded exposure, is compared to the traditional strain gauges solutions and the maintenance challenges they face. And, most importantly, the exciting new opportunities that the new measurement units can offer, are analysed, evaluating the re-purpose capacity of the health monitoring systems fibre optic sensors as sustainable real time operational monitoring systems. The originally designed structural sensors are proved to be useful also to detect, count and classify operational traffic using the infrastructure, by applying machine learning techniques that add another benefit of the use of these type of measurement sensors into the infrastructure's world.

\section{Notation}

AADT: Average Annual Daily Traffic CNN: Convolutional Neural Networks LSTM: Long Short-Term Memory HMS: Health Monitoring System

\section{Introduction}

In the year 2000 one of the first Fibre Bragg Grating (FBG) Sensors arrays were installed embedded in the bridge structure in Spain. The engineering company behind the project was APIA XXI (later Louis Berger IDC - APIA XXI, nowadays part of WSP Global Inc.), and the technology and implementation was performed by the Photonics Engineering Group at the University of Cantabria.

The test installation was done within the reinforced concrete infrastructure on both the "Las Navas" bridge, and a smaller access bridge over the A8 highway. The fibre optic sensors, capable of measuring micro-strain and temperature changes, were designed to proof that it was possible to measure the structure deformation from within the concrete, at the end of the construction phase during the load tests.
Eighteen years later the sensors are not only still functioning within the designed parameters, but - because of the improvements on the data acquisition systems and analysis techniques - higher frequencies are possible and new uses could be applied to the measurements.

The current work is the result of several site visits investigating the survivability of the sensors, acquiring more data to analyse the risks and also the opportunities that these types of sensors offer. The technologies applied not only proved that Fibre Optic sensors can provide a valuable asset to the long-term life cycle infrastructure Health Monitoring Systems, but also that they can be re-purposed to provide operational measurements, such as detection, counting and classification of traffic making use of the infrastructure. 
Figure 1 Las Navas bridge A8 highway (Spain)

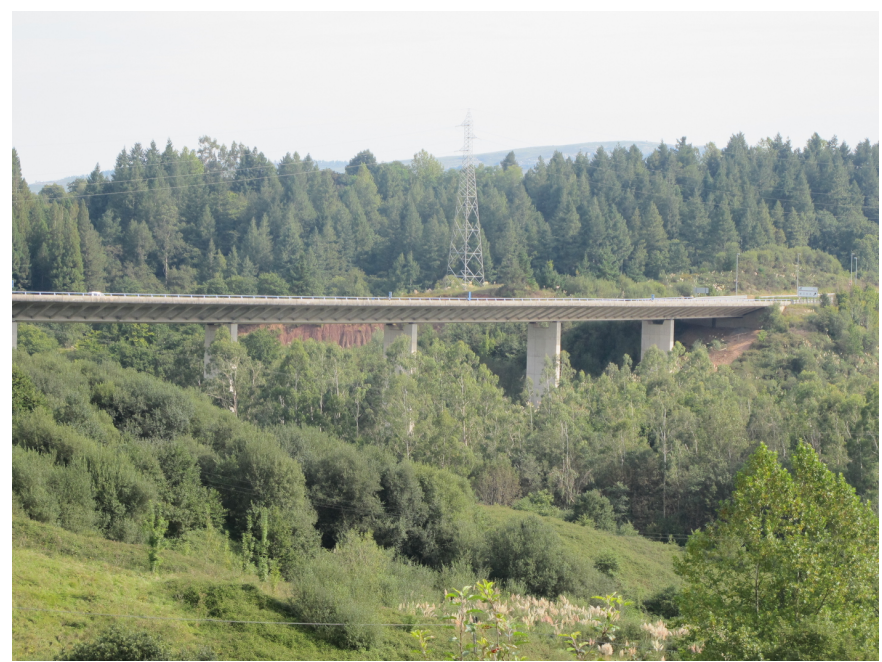

\section{Description}

\subsection{The problem}

The current exercise was triggered by the need to analyse and reduce the risks associated to long-term infrastructure health monitoring systems. The long-term lifespan of infrastructures, contrast with the short-term lifespan of most sensor technologies. New technologies are often not well proven, and therefore create an important risk regarding the survivability mismatch between the sensors used to measure and the infrastructure that needs to be measured. This mismatch is often disregarded because it is assumed to be part of the maintenance plan responsibilities, however the economical renovation of the sensors is not always possible when the structure or the measurements requires the sensors to be embedded within the material.

Many infrastructures are built with reinforced concrete, which offers a natural high compressive strength, reinforced by steel structures to compensate the low tensile strength. Apart from the normal loads they bear, these structures suffer from swelling and shrinkage, as well as thermal expansion and contraction. However, a superficial sensor will not be able to capture the required measurements due to the complex nature of the concrete, while other none invasive systems have depth and price limitations. For this application, it is clear that embedded sensor arrays are beneficial. Embedded sensors are able to measure the strain or displacement and deformation that occurs from within the structure, in a continuous way, bringing much light to the infrastructure health monitoring systems.

There are many types of embedded sensors, from the new electronic based family to the traditional wire based. The electronic based embedded sensors are very useful during construction, but they are prone to deteriorate fast with time, which creates a technology risk not acceptable for long-term infrastructure sensors. The traditional wire strain sensors require electric cables for each one of the sensors, and though there are studies supporting the long-term usage (see Simon, Alexandre et al (2015)), they offer poor scalability features. This raises the question as to which are the best and most sustainable embedded technologies to be used in Health Monitoring Systems (HMS). Is it worth investing in a certain technology for a HMS for infrastructure with a projected life of 100 years when it can be expected that half the sensors will fail within 10 years? Could the sensors be useful throughout the infrastructure life cycle, serving different purposes?

\subsection{The hypothesis}

Fibre optic methods have been available for some time, and are reaching the technology maturity required to provide reliable long-term structural health monitoring solutions. By embedding fibre optic sensors in the structures at construction time, the engineers are not only able to detect deviations from the structure's original design through time, but also allows them to measure tactical real time measurements, both useful for strategic planning and operational activities.

The adequacy debate of using Fibre Optic sensors for longterm infrastructure HMS was triggered by the recent Las Navas bridge (A-8 Autovía del Cantabrico highway, Spain) inspection site visit. The inspection, conducted by the University of Cantabria, WSP, SmartTec and Tecnalia engineers, included a successful sensor survivability survey, with some new dynamic measurements. The original objective was to check the adequacy of the long term use of such sensors for the future infrastructure health monitoring systems, but as it turned out the successful measurements captured during the site visit, and subsequent visits, triggered further analysis that explored the potential use they could offer for other purposes.

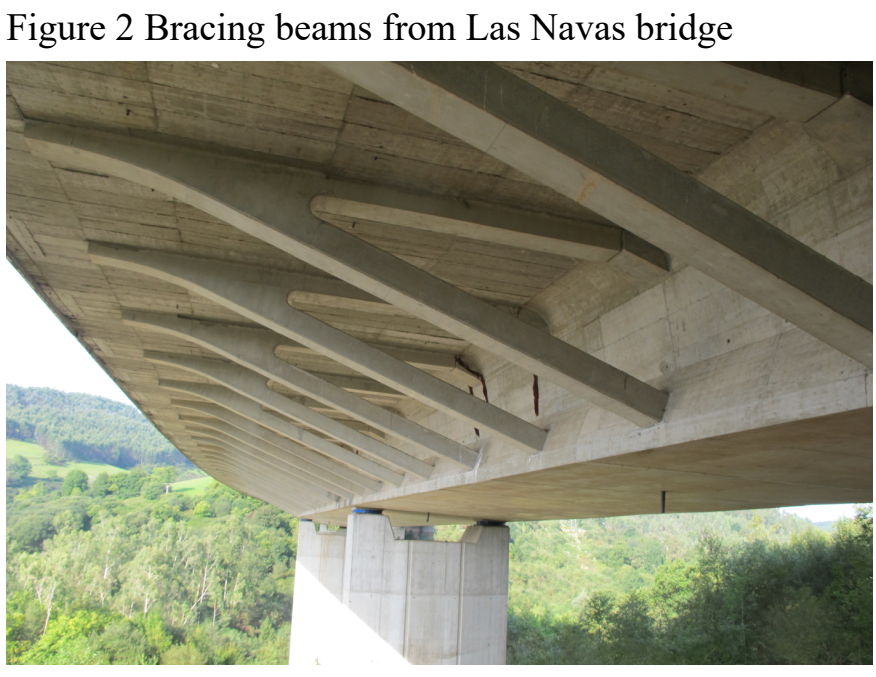

During the design and construction phase, in the year 2000, the data acquisition system available was able to take measurements from the sensors at a low $5 \mathrm{~Hz}$ frequency. This characteristic was more than adequate for the static structural measurement purposes it was designed for, able to provide the civil engineers with the low speed load test strain results they need during the construction phase. However, during the recent inspection visits, in the year 2018, the data acquisition system used was able to provide $16 \mathrm{KHz}$ frequency measurements, showing a richer dynamic dataset. The amount of dynamic measurements, and the clear correlation they had with the 
traffic using the infrastructure, raised the next assumption: if fibre optic embedded sensors survivability is long, and the measurement equipment is updated, they could offer new added value to the traffic monitoring field. The same sensors might be useful in different ways through time, making the original investment more attractive and/or increasing the resiliency of the HMS infrastructure by adding additional methods to measure its usage and demand.

This paper explores the possibility to prove that the embedded fibre optic sensors designed for static strain measurements could also be used for other dynamic operational applications, such as indirect traffic monitoring and characterization.

\subsection{The installation}

The "Las Navas" bridge is located on the A8 highway at kilometre point 250, near Cabezón de La Sal (Cantabria, Spain). It was designed and supervised by APIA XXI engineers (Louis Berger IDC, WSP Global Inc.) in the year 2000, resting over 7 piers with a curved $377 \mathrm{~m}$ long layout, with $50 \mathrm{~m}$ maximum span, and $27.6 \mathrm{~m}$ wide (see Figure 1). The design is based on a box girder resolved with a pre-stressed concrete box section. To reach the total width of the deck, there are two traverse/bracing beams every $4 \mathrm{~m}$ at each side (see Figure 2), on which the upper slab of the deck is placed, making both cantilevers $9.2 \mathrm{~m}$ long. The current configuration uses $2+2$ lanes, and serves around 19.872 AADT, composed of $88.7 \%$ light vehicles and 10.9\% heavy vehicles (2017 data).

The bridge has the singularity of having installed one of the first embedded Fibre Bragg Grating (FBG) micro-strain sensors in Spain. Other bridges around the world had FBGs installed at the time (see Tennyson (2000).), however the technology was incipient and the sensors used in this installation where designed and manufactured at the photonics laboratory from the University of Cantabria (see Jose Miguel López-Higuera (2005)). The FBGs were manufactured by exposing the germanium-doped glass fibre core to a "phase mask' under a UV radiation. The different phase masks provide a periodic refraction index grating over the fibre core, to which incident light reflects in the narrow band centred 'Bragg' wavelength. The result was packaged in an acrylic base substrate, firmly bonded or loosely enclosed - depending on the purpose of the sensor: strain or temperature FBG, and later fusion bonded to standard telecom fibre optic cable (Figure 3).

Figure 3 FBG Sensor packaging used in the structure

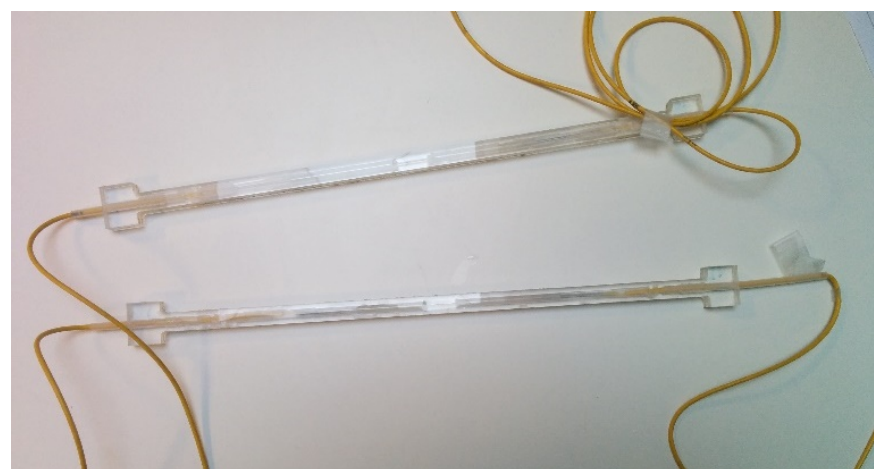

The installation was done by bonding the FBGs to the steel rebars that were embedded in concrete. The cables needed to withstand the harsh concrete or grout pouring stage as well as the curing cycle. There are reports from the time indicating that some sensors were lost during the concrete setting process. The initial count of the sensors successfully installed in the bridge were 27 FBG Strain sensors and 20 FBG Temperature sensors.

The purpose of the installation was to demonstrate the capability of the technology to measure to a high degree of precision various distributed strain points within the structure, in order to monitor the loads and structural performance during the initial settlement phase. Therefore the sensors were distributed in several fibre optic (F.O.) cables installed at key parts of the substructure (Figure 4): the box girder between two piers and over the first pier, the deck substructure, as well as within several traverse/bracing beams.

Figure 4 Sensors within the bridge structure over pier 1

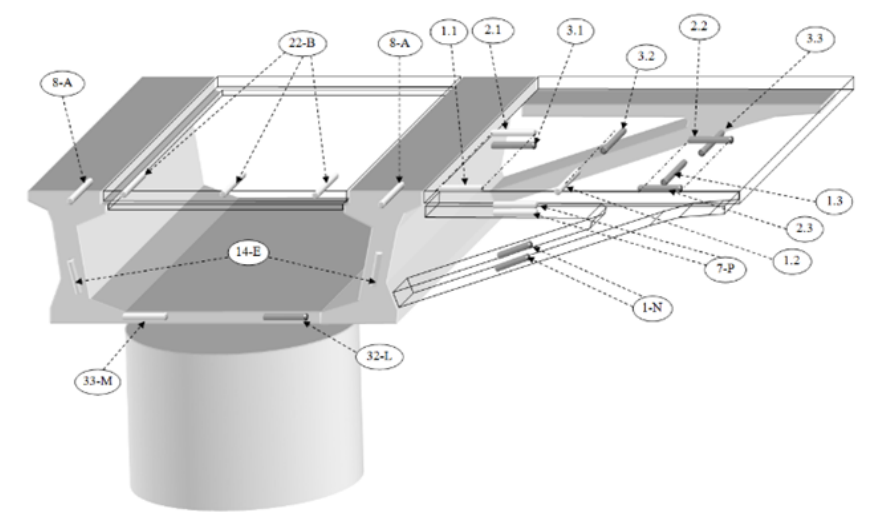

Table 1 Sensor identification (pier1)

\begin{tabular}{cll}
\hline Location & F.O. & Sensor \\
\hline Box girder & $2 \mathrm{I}$ & $32 \mathrm{~L}(\varepsilon)$ \\
& 1.1 & $1.1(\mathrm{~T})$ \\
& 1.3 & $1.3(\varepsilon, \mathrm{T})$ \\
Deck & 2.1 & $2.1(\mathrm{~T})$ \\
substructure & 2.3 & $2.2(\varepsilon), 2.3(\varepsilon, \mathrm{T})$ \\
& 3.1 & $3.1(\mathrm{~T})$ \\
Traverse/bracing & 3.3 & $3.1(\varepsilon), 3.2(\varepsilon, \mathrm{T}), 3.3(\varepsilon, \mathrm{T})$ \\
beams & $4 \mathrm{D}$ & $1 \mathrm{~N}-\mathrm{I}(\varepsilon, \mathrm{T})$ \\
\hline
\end{tabular}

\section{Procedure}

\subsection{Data acquisition}

After receiving the appropriate permits from the asset management (General Road Management department of the Ministry of Public Works), the first site visit used a FBG interrogator based on Ibsen I-MON Interrogation Monitor, with $16 \mathrm{KHz}$ measuring frequency. 
As a result of the first visit we concluded that more than $98 \%$ of the originally working FBG sensors had survived, and are able to provide comparable strain and temperature measurements readings after 18 years of being embedded on the structure. The not reachable FBG might have suffered a fibre cut or a detachment from the substrate that leaves it unusable.

Due to the FBGs high sensitivity and the higher frequency interrogation unit, it is possible to distinguish heavy vehicle traffic in real time. This triggered the discussion about the potential of the readings to monitor traffic, or even heavy vehicle load estimations.

Following the discussions, the authors organized two more site visits in order to record both FBG measurements and traffic at the same time. The result was a dataset composed of strain and temperature FBG sensor readings for several F.O. and traffic video feeds in which it was possible to identify the characteristic of the vehicles using the infrastructure.

Sensors $1 \mathrm{~N}-\mathrm{I}$ and $1 \mathrm{~N}-\mathrm{S}$ are located in the bracing beans, therefore working in compression, and showing value shifts proportional to the vehicle location, load and speed.

Table 2 4I/4D F.O. Sensor parameters and wavelength

\begin{tabular}{lll}
\hline Sensor & Parameter & Wavelength \\
\hline \hline $1 \mathrm{~N}-\mathrm{I}(\varepsilon, \mathrm{T})$ & Strain & $1556.5 \mathrm{~nm}$ \\
& Temperature & $1543.5 \mathrm{~nm}$ \\
$1 \mathrm{~N}-\mathrm{S}(\varepsilon)$ & Strain & $1549.1 \mathrm{~nm}$ \\
\hline
\end{tabular}

(Sensors within the bridge structure over pier 1)

Heavy vehicles are easily detected, even showing often a difference between the tractor head and the trailer. It is also possible to detect the nearby expansion joint vibration, after the vehicles leave the deck of the bridge. Light vehicles can also be detected, above all if they go over the slow lane. However the signal from motorcycles and other light vehicles running over the fast lane (further away from the transversal beans) is not clearly distinguishable from the background noise.

Figure 5 Measurement example sensor 1N-S

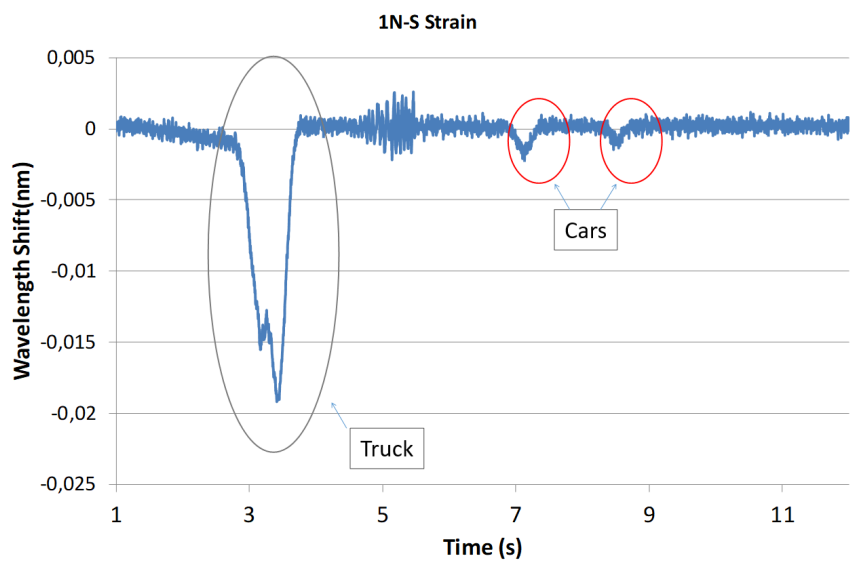

\subsection{Methodology}

Traditional signal analysis techniques require extensive data preparation and specific processing to develop the data models that provide the wanted results. However, the current exercise was intended to verify the capabilities of the sensors to provide other valuable data, without the need to analyse the specific physics of each sensor (location, compression and expansion behaviour, delays, etc...). For this purpose the use of black box neural networks techniques seem to be adequate for the analysis.

The data required to use neural networks can be described as a big amount of measurements with "features" (variables) and "labels" (results). Considering the FBGs measurements as "features", the traffic characteristics from the video recordings would be the "labels". Therefore the first task involved analysing the video recordings to extract the traffic characteristics of the infrastructure users.

To perform the label analysis different techniques where applied: visual inspection, single line image detection (as a simplified barrier analysis), and a fast pre-trained Convolutional Neural Network called YOLO (Joseph Redmon (2016)) to detect and classify vehicles from the video recordings.

The feature pre-processing included temperature compensation and noise filtering procedures. Also k-Means and hierarchical unsupervised learning clustering techniques were applied. These techniques consist of finding subgroups or structures within a given data set. The aim is to simplify the data by finding subsets of homogeneous observations within the FBG measurements, to test their self-discrimination capabilities.

Since the temporal aspect of the measurements is of high importance to the objective, the complete dataset analysis was performed using Recurrent Neural Networks (RNN) with Long Short-Term Memory cells (LSTMs). The LSTM require little dataset feature pre-processing to analyse time series information.

\section{Analysis}

\subsection{Traffic label analysis}

The traffic video recording analysis required identifying time (when), place (lane) and type of vehicle that circulated over the sensorized structure parts. The vehicle categories included eight heavy vehicles and three light vehicle types.

The visual inspection was regarded as a time consuming but necessary quality-checking method. The initial approximate synchronization and indexing with the measurements was performed matching a reduced image obtained from a single line detection-per-frame with the sensor measurements. The analysis effectively worked as a simplified barrier analysis (see Figure 6). 
Figure 6 Traffic image example using single line detection.

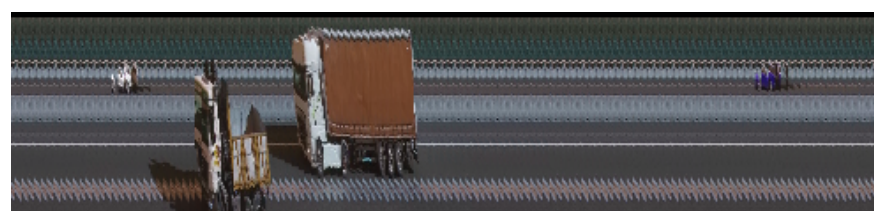

For the characterization a fast pre-trained Convolutional Neural Network called YOLO (Joseph Redmon (2016)) was used. The results were corrected with the visual inspection and provided as the vehicle classification labels for the measurements (see Figure 7).

Figure 7 Vehicle detection example over pier 1

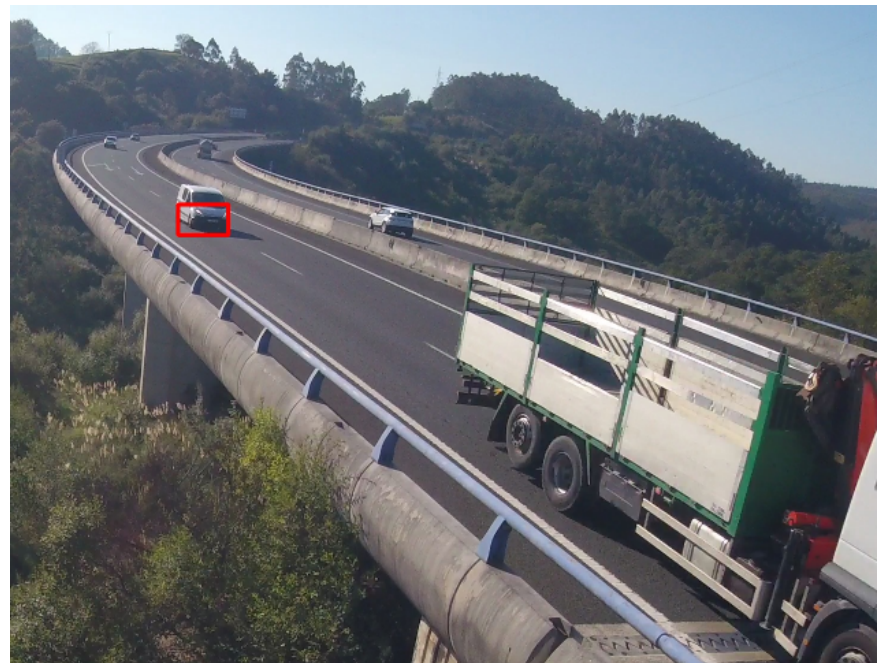

\subsection{Feature pre-processing and analysis (clustering)}

The initial analysis of a measurement dataset example (Figure 7) showed the effect of a truck passing over the bridge structure at high speed. Since some of the sensors are placed in the direction of the traffic flow, and others are orthogonal, there is a variety of effects that can be detected. The clearest strain measurement over time shows a "W" shape measurement, with the initial traction peak, followed by a stress valley and a final traction peak. Some other strain measurements are highly correlated but in a negative way ("M" shaped) due to their location. Therefore one of the first pre-processing tasks is to normalize the data.

If we observe the measurements for a longer period (see Figure 10) we can see how the strain FBGs (1556 and 1549nm) show perturbance that correspond with: the ongoing traffic effect on the structure. However, they also show a periodic compensation mechanism from the interrogation unit and a long term drift (more visible on the $1556 \mathrm{~nm}$ FBG). Subtracting the temperature readings from the strain measurements we can compensate the periodic mechanism.

Taking away the effect of the periodic compensation mechanisms from the interrogation unit adds a little noise to the strain measurements, which can be cleaned by finding the moving average for every 30 milliseconds (time that takes a $120 \mathrm{Km} / \mathrm{h}$ vehicle to move 1 meter).

The drift on time could be compensated and flattened, but seems not to be of importance for the objective of this research (furthermore if an online embedded sensor is to be developed in the future, not needing this compensation would allow to reduce the memory requirements).

Another pre-processing technique applied was data memory size reduction in order to improve the computational time.

Figure 8 FBG Measurements of 1N-S\&1N-I (15 minutes)

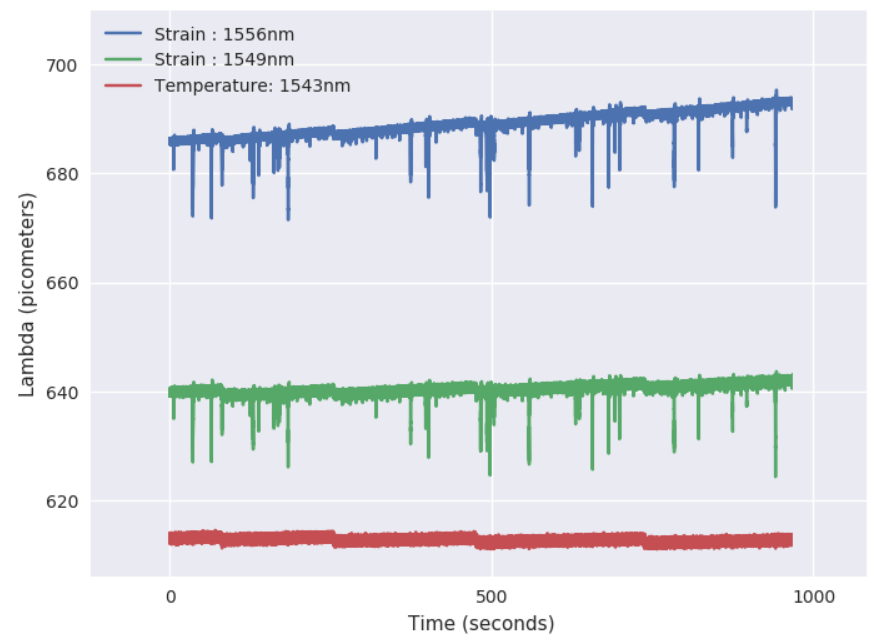

The research included applying clustering techniques like kmeans. This algorithm allows data to be grouped into $\mathrm{K}$ clusters without intersections, given the number K.

When using K-means the results show that it is easy to differentiate the signal sections, regardless of the seed used (Kmeans with $\mathrm{k}=3$ with different seeds). However, $\mathrm{k}$-means does not work like a simple threshold, but instead tries to minimize the distance function between the clusters, therefore if performing the same test over and extended dataset (longer notraffic measurements), or repeated signals, it is not identifying the signals as one cluster, but instead as different parts or continuous slopes. When analysing which seems to be the most appropriate cluster number to use for the given measurement, we get that for this case $\mathrm{k}=3$ or 4 explains the majority of withinss (Within groups sum of squares). 
Figure 9 Clustering attempt for a FBG Measurement
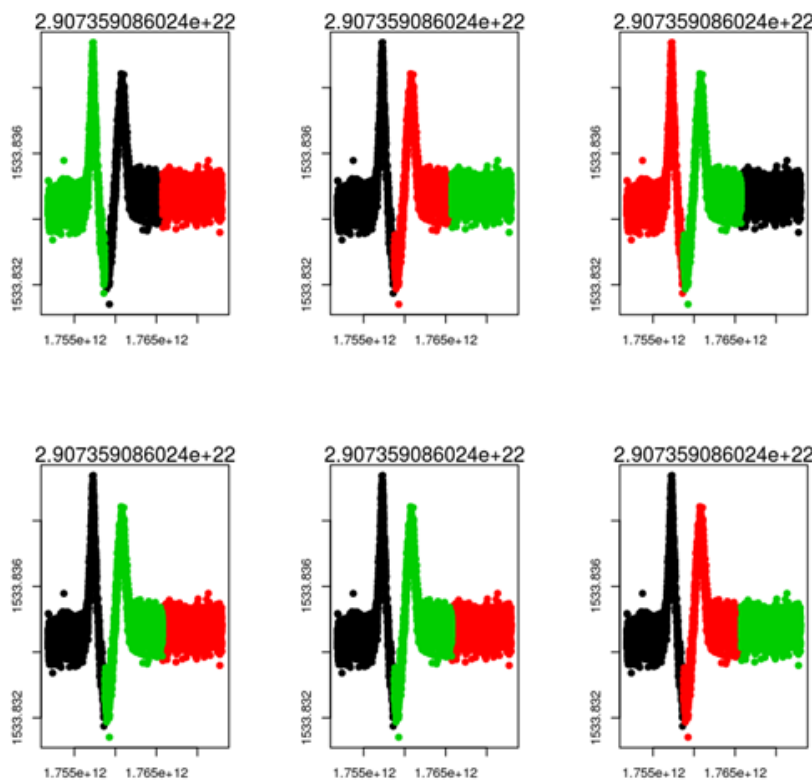

When using the hierarchical clustering, and comparing "time" and "wavelength1", it does not yield any significant information, however, if "wavelength1" and "wavelength2" are compared, it can be used to confirm that 3 is a good and balanced cluster size for the original dataset. When exploring different inter-clustering linkage techniques, the results show how "Complete, Average and Centroid" techniques are more balanced, but the "Single linkage" shows a simpler approach since it uses the minimum distance between points belonging to two different clusters, and the measurements of the dataset are time correlated. However, when comparing different wavelengths ("wavelength1" and "wavelength2") the "Complete" linkage method shows a balanced dendrogram, but the "Average, Centroid and Single" linkage methods show little discrimination capabilities.

Using MCLUST function and the k-Mean results as labels, we tried to compare the observation and prediction of the two methods, detecting that the accuracy is low, since the results are significantly different.

When using SOM clustering, with both rectangular and hexagonal topologies, the results are quite different from KMeans, but of course similar between them (just needing to correct the cluster names).

The last exercise was to try to reduce the dimensionality with principal components performed by estimating new labels, based on a threshold level of wavelength change for Wavelength1. If we repeat the measurement six times randomly and consider the variation of less than $0.003 \mathrm{~nm}$ or more than $0.006 \mathrm{~nm}$, then we have labels 1,2 and 3 (low, average and high deviation). The result was that due to the limited amount of variables it is not feasible to reduce the dimension.

\subsection{Dataset analysis with LSTM NN}

Recurrent Neural Networks (RNN) with Long Short-Term Memory cells (LSTMs) is a type of Artificial Neural Network that can process sequential data/time series. It requires to extract 'time series feature vectors' to convert them to a 'probability vector' at the output for classification.

Taking into account that each sample represented $1.2 \mathrm{~ms}$, the time series tested during the research analysis included 2600 time series feature vectors samples, 800 samples and 600 samples (with 1250, 400 and 300 overlap respectively). The wider 2600 batch was chosen with the criteria to allow the expansion joint vibration to be analysed together with a slow truck perturbance, however the long computational times and not significant results suggested to reduce the time series feature vector sizes.

The dataset was divided in a train dataset $(80 \%)$ and a test dataset (20\%). With two input features (FBGs), and starting with a unique set of three output labels: "no vehicle, light vehicle or heavy vehicle", and increasing them to the rest. Using the tensorflow LSTM (RNN) with two stacked LSTM cells (see Chevalier(2016)).

\section{Figure 10 FBG signal and labels}

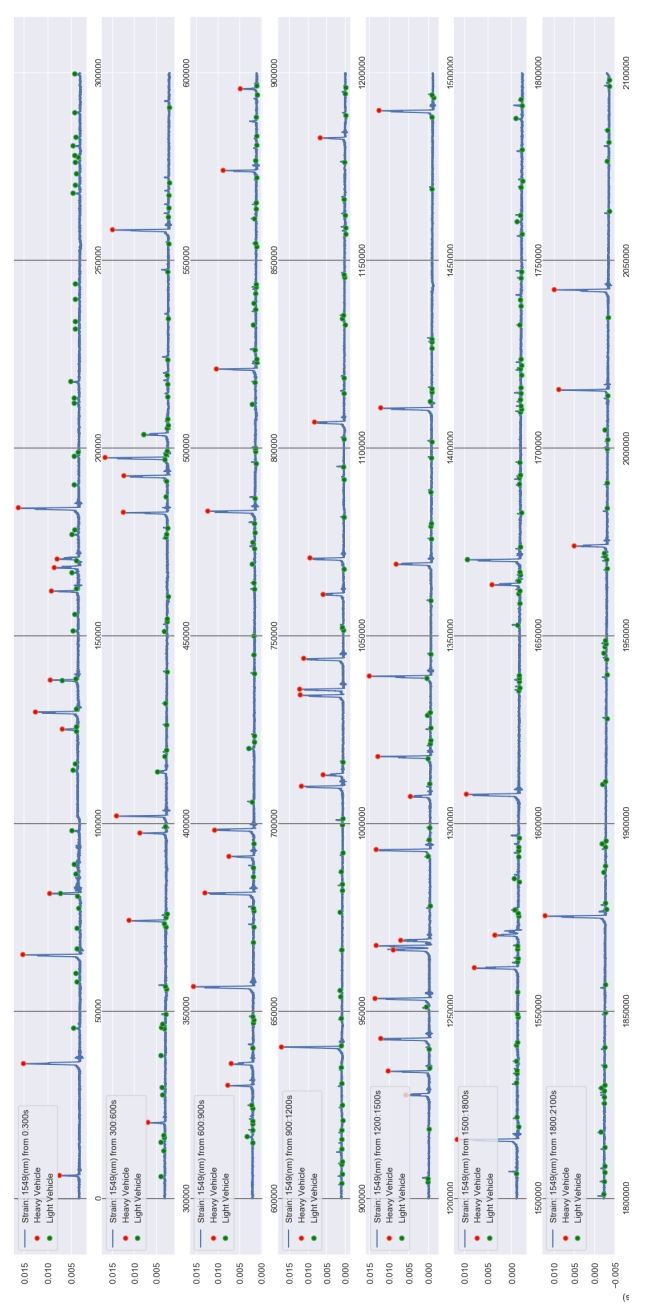




\section{Results}

The final accuracy of $89 \%(0.8901546001434326)$ indicates the neural network is almost always able to correctly identify between three output labels (see Figure 11 and 12). However when analysing the results with a wider set of vehicle classes the results are less impressive (0.6175796747207642). The main reasons are related to the difficulty to distinguish some light vehicles traveling over the fast lane at the same time, confusing the vehicles with the background noise.

Figure 11 Training iteration Loss and Accuracy

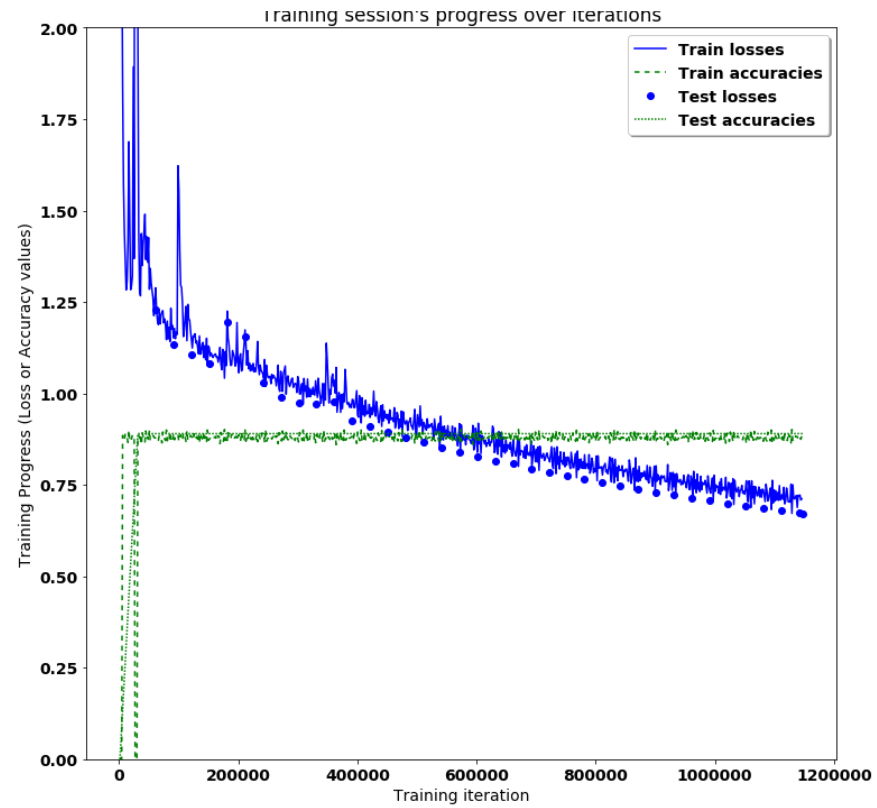

Figure 12 Confusion matrix

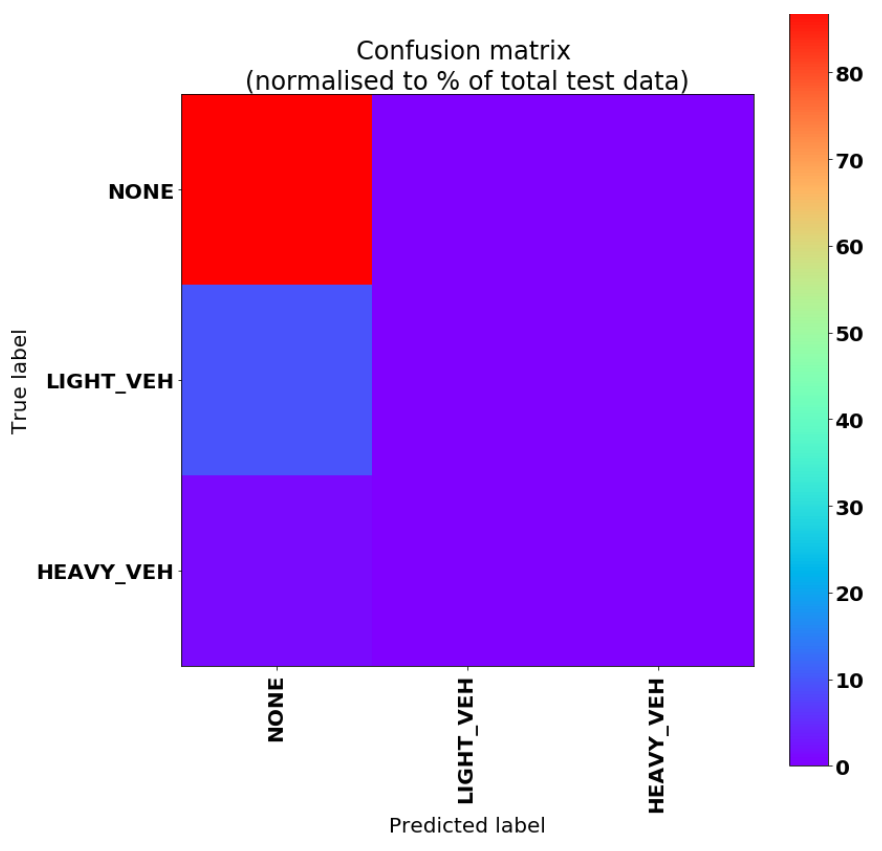

\section{Conclusion}

After 18 years fibre optic sensors seem to have become of age. They have reached a very attractive maturity level, in which they have proven not only that they can last when embedded within the infrastructure concrete, but that they can continue to offer potential to new applications.

The machine learning techniques can help finding new repurposed uses to the measurements. The results are promising and should improve when adding more accurate measurements and features.

The future work should target increasing the dataset size with more monitoring hours and different synchronized sensor measurements would increase the feature dimension and provide better discrimination.

It would be convenient to include in the FBG operational installation procedures an Optical time-domain reflectometer (OTDR) survey, to leave with the infrastructure records for reference, in order to allow future troubleshooting and comparison analysis.

\section{Acknowledgements}

To all members, partners and colleagues that helped, enabled and contributed, especially to Luis Rodriguez and Adolfo Cobo from the Photonics Engineering Group, of the Electronics Technology, Systems and Automation Engineering Department at the University of Cantabria, Lara Lloret and the teachers at the Master of Data Science coordinated by the IFCA. Juan Carlos Salcines, Antonio Herrera and Mariano Rodriguez from the Engineering Company Louis Berger IDCAPIAXXI (WSP Global Inc). The fibre sensor company SMARTEC and also the R\&D H2020-EU.3.4. project RAGTIME (Grant agreement ID: 690660), thanks to which it all started with the first site visit.

\section{References}

\section{Journal articles}

Tennyson RC et al (2000). Structural health monitoring of innovative bridges in Canada with fiber optic sensors, 2001 Smart Mater. Struct. 10560 (http://iopscience.iop.org/09641726/10/3/320)

\section{Conference paper}

Simon, Alexandre \& Courtois, Alexis \& Clauzon, Timothée \& Coustabeau, Eric \& Vinit, Simon. (2015). Long-term measurement of strain in concrete: durability and accuracy of embedded vibrating wire strain gauges. Conference: SMAR 2015, At Antalya, Turkey.

Joseph Redmon et al (May 2016) You Only Look Once: Unified, Real-Time Object Detection (YOLO) University of Washington. https://arxiv.org/pdf/1506.02640.pdf

Joseph Redmon, Ali Farhadi (Dec 2016) YOLO9000: Better, Faster, Stronger. University of Washington. https://arxiv.org/pdf/1612.08242.pdf 


\section{Theses}

Cobo, L. (2013): Optical sensors for smart structures, PhD thesis Universidad de Cantabria, Spain. p.103 Pre-stressed concrete beam monitoring.

\section{Websites}

Website as main reference

Guillaume Chevalier, LSTMs for Human Activity Recognition, 2016, https://github.com/guillaume-chevalier/LSTM-HumanActivity-Recognition

Website as further information

Viaducto sobre el Arroyo de Las Navas. See http://www.robertorevillaestudio.es/viaducto las_navas.html for further details (accessed 20/01/2019)

Traffic statistics for 2017. See https://www.fomento.es/recursos mfom/comodin/recursos/m approvin 2017.pdf for further details (accessed 21/12/2018) 